\section{MP25-15 \\ INITIAL PHARMACOTHERAPY FOR OVERACTIVE BLADDER SYMPTOMS AMONG MEDICARE BENEFICIARIES}

Charles Scales Jr*, Melissa Greiner, Lesley Curtis, Brad Hammill, Andrew Peterson, Cindy Amundsen, Viviana Martinez-Bianchi, Mitchell Heflin, Kenneth Schmader, Durham, NC

INTRODUCTION AND OBJECTIVES: Overactive bladder (OAB) affects up to $40-50 \%$ of older men and women. OAB diminishes quality of life through its impact on daily living and emotional well-being, even in the absence of urine loss. The economic costs of treating patients with $O A B$ are projected to exceed $\$ 80$ billion annually by 2020 , more than half incurred by Medicare beneficiaries. Despite this burden of disease, no prior study has examined pharmacotherapy prescribing patterns in the Medicare population. Our objective was to describe variation in initial drug treatment of $O A B$ symptoms among Medicare Part $D$ beneficiaries.

METHODS: We performed a cross-sectional analysis of beneficiaries using the Medicare 5\% sample. Between 2007-2013, we identified beneficiaries with an outpatient encounter consistent with $\mathrm{OAB}$ symptoms and a Medicare Part $D$ drug claim for a first generation (pre-2000 FDA approval: oxybutynin, tolterodine) or a second generation (post-2000 FDA approval: darifenacin, fesoterodine, mirabegron, solifenacin, trospium) agent. Subjects with urinary tract infection within 30 days, neurogenic bladder, and prostate cancer diagnoses were excluded. No subject had a prior OAB drug claim. We used a multivariable log-binomial regression model to estimate the relative probability of receipt of an initial 2nd generation agent, controlling for beneficiary demographics, comorbidity, provider specialty, and geographic variation.

RESULTS: During the study period, 17,944 beneficiaries had an initial drug claim. The average subject was aged $78 \pm 7.5$ years, $80 \%$ were female, and $89 \%$ were white. Beneficiaries dually eligible for Medicaid constituted $26 \%$ of the cohort. In multivariable models, black beneficiaries and dual-eligibles were slightly less likely to receive 2 nd generation agents as first line therapy (black: RR 0.91, $p=0.015$; Medicaid: RR 0.95, $p=0.012$ ). Urologists and gynecologists were substantially more likely than generalists to prescribe 2 nd generation drugs (urology: RR 1.49, $p<0.001$; gynecology: RR 1.30, $p<0.001$ ). Subjects with dementia were $13 \%(95 \% \mathrm{Cl} 6 \%-19 \%)$ more likely to receive a 2 nd generation agent.

CONCLUSIONS: Medicare beneficiaries initially treated by specialists are substantially more likely to receive 2 nd generation $O A B$ agents as first line therapy. While side effect profiles may differ, current guidelines do not support substantial efficacy differences among these agents. Further investigation is required to understand differences in outcomes and costs associated with these disparate prescribing patterns.

Source of Funding: NIH/NIA GEMSSTAR program (R03AG048130) and American Geriatric Society Dennis W. Jahnigen Career Development Award (Scales).

\section{MP25-16 \\ MEDICATION SWITCHING AFTER INITIAL PHARMACOTHERAPY FOR OVERACTIVE BLADDER}

Charles Scales Jr*, Melissa Greiner, Lesley Curtis, Brad Hammill, Andrew Peterson, Cindy Amundsen, Viviana Martinez-Bianchi, Mitchell Heflin, Kenneth Schmader, Durham, NC

INTRODUCTION AND OBJECTIVES: Overactive bladder $(\mathrm{OAB})$ affects nearly half of older men and women. Anti-cholinergic medications are a mainstay of treatment, yet often have only moderate efficacy and potentially substantial side effects. These side effects are particularly concerning for older adults and may lead to switching among drug agents in pursuit of greater efficacy or fewer side effects. Despite this important clinical problem, no prior study has examined drug switching patterns in the Medicare population. Our objective was to describe variation in medication switching within 6 months of initial pharmacotherapy for $\mathrm{OAB}$ symptoms among Medicare Part $D$ beneficiaries.

METHODS: We performed a retrospective cohort study of beneficiaries using the Medicare 5\% sample. Between 2007-2013, we identified subjects with an outpatient encounter consistent with $\mathrm{OAB}$ symptoms and a Medicare Part $D$ drug claim for a first generation (pre2000 FDA approval: oxybutynin, tolterodine) or a second generation (post-2000 FDA approval: darifenacin, fesoterodine, mirabegron, solifenacin, trospium) agent. No subject had a prior OAB drug claim. We followed subjects for 6 months after the initial claim, and defined switching as any new prescription for a different OAB-directed agent. We used a multivariable log-binomial regression model to estimate the relative probability of medication switch, controlling for beneficiary demographics, comorbidity, and geographic variation.

RESULTS: During the study period, 15,923 beneficiaries had Part $D$ enrollment for at least 6 months after the index drug claim. The average subject was aged $78 \pm 7.5$ years, $80 \%$ were female, and $89 \%$ were white. Tolterodine or oxybutynin constituted $56 \%$ of initial prescriptions. Within 6 months, 2,457 (15\%) switched agents. In multivariable models, females were $25 \%$ more likely to switch, as were nursing home residents (RR 1.29, $\mathrm{p}<0.001)$. Switching was also more likely when the prescribing physician was a urologist $(R R 1.18, p<0.001)$ and when the initially prescribed agent was a second generation medication. In sensitivity analyses, out-of-pocket patient cost was not associated with medication switching. The only initial agent not associated with switching within 6 months was mirabegron.

CONCLUSIONS: Switching is common among Medicare beneficiaries treated with medication for OAB symptoms. Changing prescriptions is associated with patient characteristics, provider and initial agent, but not patient cost. Further investigation is required to understand drivers and outcomes of these switching patterns to improve care in this vulnerable population.

Source of Funding: NIH/NIA GEMSSTAR program (R03AG048130) and American Geriatric Society Dennis W. Jahnigen Career Development Award (Scales).

\section{MP25-17 \\ CONTEMPORARY UTILIZATION TRENDS OF SYSTEMIC CHEMOTHERAPY FOR UPPER TRACT UROTHELIAL CARCINOMA (UTUC)}

Mohammed Haseebuddin*, Elizabeth Handorf, Joshua Jones, Alexander Kutikov, Nikhil Waingankar, Benjamin Ristau, Michael Haifler, Yu-Ning Wong, Rosalia Viterbo, Richard Greenberg, Robert Uzzo, David Chen, Marc Smaldone, Philadelphia, PA

INTRODUCTION AND OBJECTIVES: There is currently no consensus regarding the use of systemic therapy for surgically-treated patients with invasive UTUC. Using a large national cancer registry, our objective was to assess temporal trends in utilization of systemic therapy for stage II-IV UTUC.

METHODS: The National Cancer Database (NCDB) was queried for all patients surgically treated (nephroureterectomy, segmental resection) for stage II-IV UTUC from 1998-2012. Temporal trends in receipt of systemic therapy [neoadjuvant (NAT), adjuvant (AT), or unknown timing (UKT)] were assessed using chi square analyses. After exclusion of patients receiving NAT or UKT, adjusting for patient and clinicopathologic characteristics, multivariable logistic models were used to examine the association between clinicopathologic characteristics and receipt of AT. Kaplan Meier analyses were performed comparing overall survival (OS) between patients receiving AT and those who did not.

RESULTS: Of 7,629 patients identified over the study period, $24.1 \%$ of patients surgically treated for stage II-IV UTUC received any systemic therapy (NAT: $1.44 \%$, AT: $19.11 \%$, UKT: $3.51 \%$ ). Utilization of any systemic therapy significantly increased from $1998-2012(20.2 \%$ vs. $28.7 \%, p<0.0001)$. Following adjustment, patients of increased age 